\title{
Athermal annealing of Si-implanted GaAs and InP
}

\author{
Mulpuri V. Rao,a) J. Brookshire, and S. Mitra \\ Department of Electrical and Computer Engineering, George Mason University, Fairfax, \\ Virginia 22030-4444
}

Syed B. Qadri

Materials Science and Technology Division, U.S. Naval Research Laboratory, Washington, DC 20375

R. Fischer and J. Grun

Plasma Physics Division, U.S. Naval Research Laboratory, Washington, DC 20375

\section{N. Papanicolaou}

Electronics Science and Technology Division, U.S. Naval Research Laboratory, Washington, DC 20375

M. Yousuf

George Washington University, Washington, DC 20052

M. C. Ridgway

Department of Material Science and Engineering, Australian National University, Canberra, ACT 0200, Australia

(Received 2 October 2002; accepted 31 March 2003)

\begin{abstract}
GaAs and InP crystals ion implanted with $\mathrm{Si}$ were athermally annealed by exposing each crystal at a spot of $\sim 2 \mathrm{~mm}$ diameter to a high-intensity $1.06 \mu \mathrm{m}$ wavelength pulsed laser radiation with $\sim 4$ $\mathrm{J}$ pulse energy for $35 \mathrm{~ns}$ in a vacuum chamber. As a result a crater is formed at the irradiated spot. The crater is surrounded by a dark-colored ring-shaped region which is annealed by mechanical energy generated by rapidly expanding hot plasma that formed on the exposed spot. The electrical characteristics of this annealed region are comparable to those of a halogen-lamp annealed sample. No redistribution of impurities due to transient diffusion is observed in the implant tail region. In $\mathrm{x}$-ray diffraction measurements, a high angle side satellite peak due to lattice strain was observed in the crater and near crater regions of the athermally annealed sample in addition to the main Bragg peak that corresponds to the pristine sample. This high angle side satellite peak is not observed in regions away from the crater ( $\geqslant 5 \mathrm{~mm}$ from the center of the crater in GaAs). (C) 2003 American Institute of Physics. [DOI: 10.1063/1.1576896]
\end{abstract}

\section{INTRODUCTION}

Ion implantation is the most widely used technique to achieve selective area doping of compound semiconductors like GaAs and InP. ${ }^{1,2}$ This is because thermal dopant diffusion technology is either unreliable or not possible for these materials due to their low incongruent evaporation temperature. Although doping by ion implantation is an attractive alternative, this process introduces lattice damage and the implanted dopant atoms mostly occupy electrically inactive interstitial lattice positions. Annealing is necessary after ion implantation in order to remove lattice damage and to drive implanted dopant atoms into electrically active substitutional lattice sites. The donor, (e.g., Si) and acceptor, (e.g., Mg) dopants of interest in $\mathrm{GaAs}$ and $\mathrm{InP}$ do not contribute to electrical conduction when they reside in interstitial positions, which they normally assume in the as-implanted state. ${ }^{1}$

At present, annealing of GaAs and $\mathrm{InP}$ is primarily performed using thermal processes such as quartz-tube-furnace annealing or halogen-lamp rapid thermal annealing (RTA). The main problems with such thermal annealing techniques include impurity diffusion during annealing, ${ }^{1}$ the creation of new types of defects, and incongruent evaporation of the

${ }^{a)}$ Electronic mail: rmulpuri@gmu.edu substrate material. Impurity diffusion during annealing is undesirable for submicron devices, and junction leakage currents caused by the new annealing-created defects can limit the performance of the ultrahigh-density, low-power integrated circuits required for mobile communications using compound semiconductors such as GaAs and InP. Surface sublimation during annealing of these compound semiconductors limits the maximum temperature that can be used for optimum annealing and also requires the use of encapsulating layers for surface protection, adding extra, possibly complicated, processing steps. ${ }^{1,2}$

Recently, an athermal annealing (AA) technique was demonstrated to anneal neutron-transmutation-doped silicon $^{3,4}$ and phosphorus- and boron-implanted silicon ${ }^{5,6}$ over small regions. Annealing by this technique is faster than thermal annealing and avoids the direct application of thermal energy. In AA, high-power laser pulse radiation is focused onto a small spot on an implanted semiconductor wafer. This can initiate a process which electrically activates implant dopants and removes implantation-induced lattice damage in regions far beyond the laser-illuminated spot, where not much thermal energy heat is directly available. ${ }^{3,4}$ This technique is different from conventional pulsed laser annealing, in which a relatively low power density laser pulse (focused to a few $\mathrm{J} / \mathrm{cm}^{2}$ ) is used to heat and anneal 
areas of the wafer directly beneath the laser spot and is rastered over the wafer surface to anneal the entire implanted area. In the AA technique, a laser pulse focused to a much higher intensity $\left(\sim \mathrm{a}\right.$ few $\left.\mathrm{kJ} / \mathrm{cm}^{2}\right)$ at just one spot is expected to anneal implanted regions far away from the spot. In this case, annealing is not due to bulk heating because the laser pulse focused onto a small spot does not have sufficient energy to heat regions far away significantly. Variation of the temperature with the radial distance and time has been estimated using the classical Landau and Lifshitz model. ${ }^{7}$ Using the physical constants of GaAs and the equation $T(r, t) \alpha(\chi t)^{-1 / 2} \exp \left(-r^{2} / 4 \chi t\right)$, where $T, r$ and $t$ are the temperature, distance and time, respectively, and $\chi=\kappa / \rho c_{p}$ is the thermal conductivity divided by the density and specific heat at constant pressure, we found that the temperature decreases by at least three orders of magnitude $1 \mathrm{~mm}$ from the illuminated spot. The validity of this equation has been experimentally verified for $\mathrm{Si}$ by Grun $e t$ al. ${ }^{8}$ using an array of photoresist rectangular line patterns on a $\mathrm{Si}$ wafer subjected to pulsed laser radiation. According to the experimental results, the temperature falls rapidly with the distance and hence no significant heating is expected outside the illuminated spot. The optical energy is absorbed by plasma above the sample surface. When the plasma gets very hot it expands precipitously and generates recoil pressure, which drives shock and sound waves into the regions surrounding the laser-exposed spot. ${ }^{4}$

In this work, silicon-implanted GaAs and InP were subjected to the athermal annealing technique to explore its potential use in annealing implanted III-V compound semiconductors for use in high-frequency and optoelectronic devices. The annealed material is characterized for its electrical, implant thermal stability and lattice perfection characteristics.

\section{EXPERIMENT}

Multiple-energy (30-350 keV) silicon ions were implanted into semi-insulating GaAs and InP substrates at room temperature to obtain uniformly doped box profiles of $\sim 0.4$ $\mu \mathrm{m}$ depth at concentrations of $1 \times 10^{19}, 5 \times 10^{18}$ and 5 $\times 10^{17} \mathrm{~cm}^{-3}$ for GaAs and $2 \times 10^{19}$ and $5 \times 10^{17} \mathrm{~cm}^{-3}$ for InP. Silicon predominantly acts as an $n$-type impurity in GaAs and InP. These concentrations represent typical doping concentrations used for source/drain and channel regions of field-effect transistors and the crystal is not amorphized at these doping concentrations. For athermal annealing, the wafers were subjected to a single $1.06 \mu \mathrm{m}$ wavelength laser pulse of $\sim 4 \mathrm{~J}$ energy and, $35 \mathrm{~ns}$ duration, focused onto $\sim 2$ $\mathrm{mm}$ diam spot in a vacuum chamber. The samples were titled approximately $15^{\circ}$ from the normal direction toward the laser beam pulse. This helps to prevent the samples from cracking. For comparison, 950 and $875^{\circ} \mathrm{C} / 10 \mathrm{~s}$ halogenlamp RTA was performed on the implanted GaAs and InP samples, respectively. To protect the sample surface during RTA, a $50 \mathrm{~nm}$ thick $\mathrm{Si}_{3} \mathrm{~N}_{4}$ cap was used for both GaAs and InP.

Electrical characteristics of the annealed material were evaluated along the diameter of the circular annealed region surrounding the laser-irradiated spot by performing four-

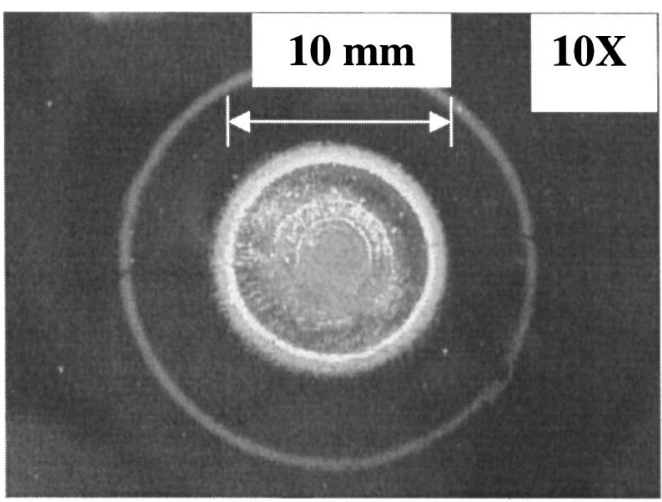

(a)

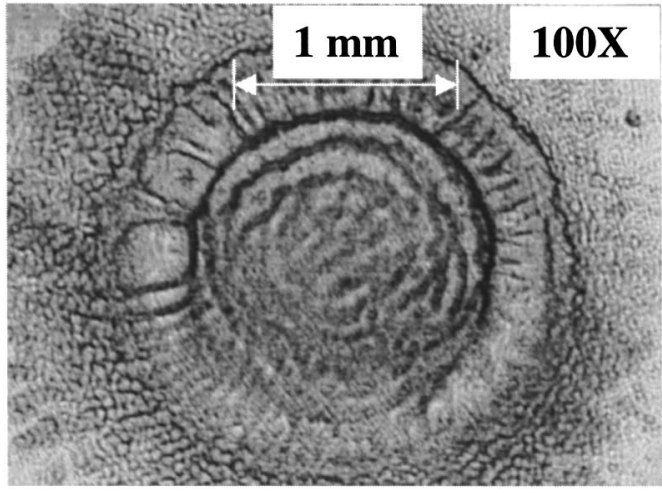

(b)

FIG. 1. Photo/micrograph of a Si-implanted GaAs sample after irradiation with a laser pulse.

point probe sheet resistance and capacitance-voltage $(C-V)$ profiling measurements. The thermal stability of the implant profile in the annealed material was evaluated by secondaryion-mass spectrometry (SIMS) measurements using a 14.5 $\mathrm{keV} \mathrm{Cs}{ }^{+}$net impact energy primary beam. Lattice perfection was evaluated by comparing the high-resolution x-ray diffraction data on pristine, as-implanted, and AA samples. High resolution $\mathrm{x}$-ray diffraction scans were obtained with a Huber four-circle diffractometer system using true $\mathrm{Cu} K \alpha_{1}$ radiation from a rotating anode $\mathrm{x}$-ray generator operated at $50 \mathrm{kV}$ and $200 \mathrm{~mA}$. The resolution of the setup is $\Delta(2 \theta)$ $=0.001^{\circ}$. Direct beam data collection indicated that the content of $\mathrm{Cu} K \alpha_{2}$ was $\leqslant 10^{-4}$.

\section{RESULTS AND DISCUSSION}

\section{A. Surface morphology}

An optical micrograph of the laser-pulse exposed region and of the surrounding annealed area is shown in Fig. 1 for Si-implanted GaAs. A crater formed at the center of the exposed spot as a result of pressure from the hot plasma produced by the high-intensity pulsed laser radiation. The crater is surrounded by an athermally annealed ring-shaped zone (called the AA inner ring hereafter) with an outer diameter of $\sim 8 \mathrm{~mm}$, which is surrounded by a partially athermally annealed ring-shaped region (called the partial AA outer ring hereafter) with an outer diameter of $\sim 16 \mathrm{~mm}$. Annealing was observed in a circular area $\sim 8 \mathrm{~mm}$ in diameter centered on 


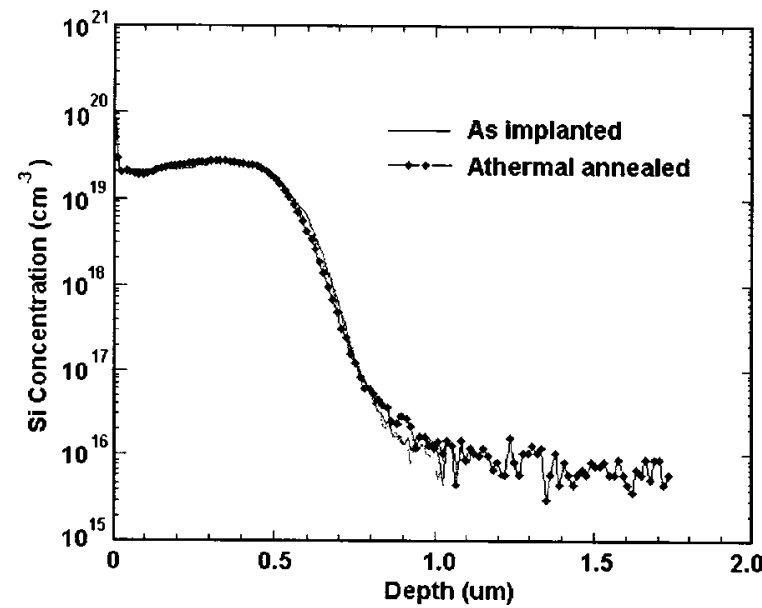

FIG. 2. Si SIMS depth profiles of Si-implanted InP before and after laserpulse athermal annealing.

the laser focal spot. In the implanted InP wafer, for the same annealing conditions, annealing was observed in a circular area of $\sim 12 \mathrm{~mm}$ diameter. A change in color was observed in the AA inner ring surrounding the crater but not in the implanted unannealed areas. The AA inner ring was dark, similar to that of a virgin GaAs crystal, suggesting removal of the implantation-induced lattice damage. A similar change in color was also seen in implanted InP irradiated by pulsed laser radiation. This implies that a high-energy laser pulse focused onto a small spot on an implanted GaAs or InP crystal can remove lattice damage induced by the implantation process in regions outside the laser illuminated spot where no heat was directly applied.

\section{B. SIMS depth profiles}

SIMS depth profiles of implanted $\mathrm{Si}$ in $\mathrm{InP}$ and after pulsed laser radiation athermal annealing are shown in Fig. 2. The implant depth profile obtained in the AA inner ring surrounding the crater is very similar to the profile in the as-implanted sample. Similar stable implant depth profile characteristics were also observed in GaAs crystals. This clearly suggests the ability of the athermal annealing technique to contain transient enhanced diffusion normally observed in the implant profile tail region of a conventional thermally annealed sample. This feature makes pulsed laser radiation athermal annealing attractive for making small dimensional GaAs and InP devices.

\section{Electrical characteristics}

Electrical characteristics of the implanted/annealed $\mathrm{GaAs}$ and InP were evaluated by performing four-point probe measurements on heavily doped $\left(\geqslant 5 \times 10^{18} \mathrm{~cm}^{-3}\right)$ samples. It is well known that at these high doping concentrations, the Schottky barrier height on $n$-type GaAs and InP is low and hence the error in resistivity measurements using the four-point probe technique is minimal. To obtain good spatial resolution, the four-point probes were placed in a configuration normal to the diameter of the circularly annealed region (i.e., in the direction parallel to the tangent of the

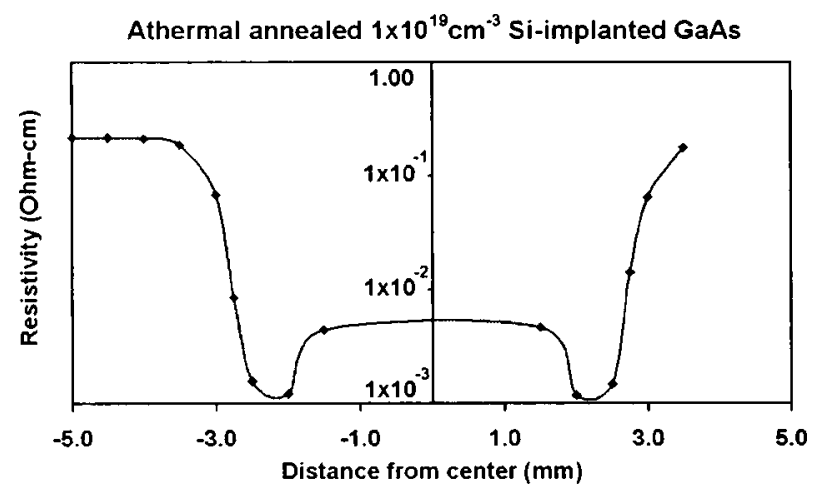

(a)

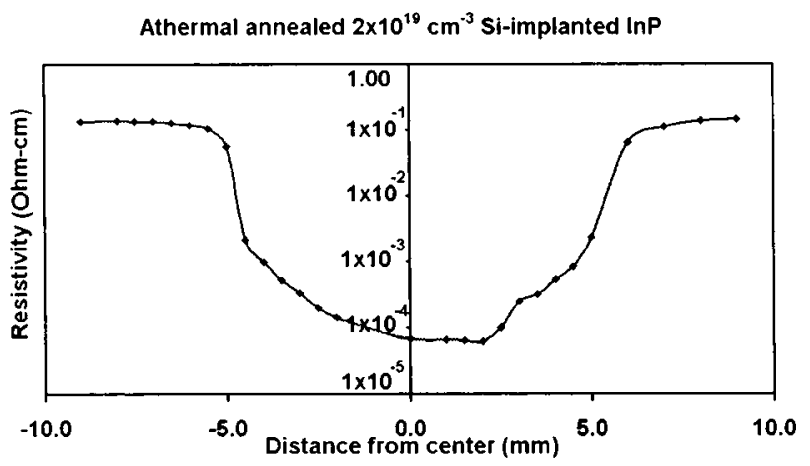

(b)

FIG. 3. Variation in resistivity along the diameter of the circularly annealed area in (a) $1 \times 10^{19} \mathrm{~cm}^{-3} \mathrm{Si}$-implanted $\mathrm{GaAs}$ and (b) $2 \times 10^{19} \mathrm{~cm}^{-3} \mathrm{Si}$ implanted InP.

circularly annealed region). In heavily doped GaAs and InP, both four-point probe and van der Pauw-Hall techniques yielded similar electrical resistivity values. Electrical resistivity, which is inversely proportional to the product of the carrier concentration and carrier mobility, is a useful quantity with which to characterize ion-implanted semiconductors. Annealing is supposed to drive implanted dopant atoms into substitutional lattice sites and result in a high carrier concentration. Annealing also results in lattice damage removal and consequently high carrier mobility. ${ }^{1,2}$ Hence, in an implanted semiconductor crystal, effective annealing results in a high carrier concentration and high carrier mobility and consequently low resistivity. In addition, conventional annealing may also introduce new defect centers in III-V compound semiconductors. ${ }^{9}$ Variation of electrical resistivity with the position from the center along the diameter of the circularly annealed area is shown in Fig. 3(a) for the GaAs crystal implanted with $1 \times 10^{19} \mathrm{~cm}^{-3} \mathrm{Si}$, and in Fig. 3(b) for the InP crystal implanted with $2 \times 10^{19} \mathrm{~cm}^{-3} \mathrm{Si}$. In the GaAs crystal, the variation in resistivity along the diameter of the circularly annealed region resembles a "W" with more effective $\mathrm{Si}$ donor activation taking place in the AA ring-shaped zone surrounding the crater. The minimum resistivity measured at a radial distance of $2-3 \mathrm{~mm}$ from the center of the crater in the AA inner ring zone in GaAs is $\sim 1.2 \times 10^{-3} \Omega \mathrm{cm}$, which closely matches resistivity of $\sim 1.3 \times 10^{-3} \Omega \mathrm{cm}$ measured in a GaAs crystal subjected to halogen-lamp RTA at $950{ }^{\circ} \mathrm{C}$ for $10 \mathrm{~s}$. At distance $\geqslant 3 \mathrm{~mm}$ from the center of the crater, the 
resistivity increased two orders of magnitude, signifying inadequate annealing in the partial AA outer ring region surrounding the dark-colored AA inner ring zone. A similar minimum resistivity value in the AA inner ring zone was observed in GaAs implanted with $5 \times 10^{18} \mathrm{~cm}^{-3} \mathrm{Si}$.

It is well known that there is minimal benefit in increasing the implanted $\mathrm{Si}$ doping concentration above mid- $10^{18} \mathrm{~cm}^{-3}$ ranges to obtain low sheet resistance because it does not result in an increase in net electron concentration. ${ }^{2,10}$ The net maximum substitutional donor concentration measured by $C-V$ profiling of Au Schottky diodes formed on $5 \times 10^{18} \mathrm{~cm}^{-3} \mathrm{Si}$ ion-implanted $\mathrm{GaAs}$ athermally annealed by a laser pulse is $2 \times 10^{18} \mathrm{~cm}^{-3}$, which is the same as the value measured in the halogen-lamp RTA material. In GaAs, the net donor concentration remains at $\sim 2 \times 10^{18} \mathrm{~cm}^{-3}$ even for higher Si implant concentrations. ${ }^{2}$

As shown in Fig. 3(b), the variation in resistivity in 2 $\times 10^{19} \mathrm{~cm}^{-3}$ Si-implanted InP along the diameter of the circularly annealed area is " $U$ " shaped with a minimum resistivity of $\sim 2 \times 10^{-4} \Omega \mathrm{cm}$ outside the crater, which is close to the value of $3.5 \times 10^{-4} \Omega \mathrm{cm}$ measured in $875^{\circ} \mathrm{C} / 10 \mathrm{~s}$ halogen-lamp annealed InP. In the InP crystal, effective annealing occurred at distances farther from the exposed spot compared to the GaAs crystal for the same annealing conditions. This could be due to differences in the material parameters. At distances $\sim 6 \mathrm{~mm}$ from the center of the crater, the measured resistivity is about three orders magnitude greater than the minimum value close to the center of the crater. This signifies inadequate annealing outside the dark-colored ring region (similar to that shown in Fig. 1). In InP, the minimum resistivity measured in heavily doped material is almost an order of magnitude less than that of GaAs, due to higher net substitutional donor activation of implanted Si in InP compared to that in GaAs. ${ }^{2}$

In both GaAs and InP, the resistivity measured in the crater region is different from the values measured in the AA inner ring zone surrounding the crater. As shown in Fig. 3, for $1 \times 10^{19} \mathrm{~cm}^{-3}$ Si-implanted GaAs, the resistivity in the crater region is higher compared to the values in the AA inner ring area, whereas for $2 \times 10^{19} \mathrm{~cm}^{-3}$ Si-implanted InP the resistivity in the crater region is lower compared to the values in AA inner ring area. The difference in resistivity values in these two regions is due to differences in the annealing mechanisms of these two regions. In the area irradiated by pulsed laser radiation the semiconductor wafer has melted in the exposed surface region and then epitaxially regrown on the unmelted bottom part of the crystal. The dark-colored AA inner ring area surrounding the crater is not melted and annealing in this area has taken place as a result of the propagation of mechanical energy generated by the plasma formed in the laser crater region irradiated by pulsed laser radiation. Thus, annealing in the AA inner ring area has taken place in the solid phase (without the material assuming, a liquid state during annealing) as in the case of halogen-lamp rapid thermal annealing. Due to this similarity in the annealing mechanisms, the electrical resistivity of the AA inner ring area is almost same as that in halogen-lamp RTA material. But due to the fundamental difference in the annealing mechanisms of the two regions, the net donor ac- tivation of an amphoteric impurity like $\mathrm{Si}$ can be different in the crater and AA inner ring areas, depending on the Si implant concentration. For $1 \times 10^{19} \mathrm{~cm}^{-3} \mathrm{Si}$ in GaAs, the amphoteric incorporation of $\mathrm{Si}$ in lattice sites may occur at a higher level in the crater region compared to in the darkcolored AA inner ring region and result in a lower net donor concentration and consequently higher resistivity in the crater region. X-ray diffraction measurements indicate more residual implant damage in the crater region compared to in the AA inner ring area, which is expected to result in low carrier mobility and consequently in high resistivity. Since the laser induced melted zone is splat quenched, the lattice does not relax fully in the crater region. Hence, remanent compressive stress is observed in the crater region. For $\mathrm{GaAs}$, the degree of amphoteric behavior of implanted $\mathrm{Si}$ in the crater region is also a function of the Si concentration. For the $5 \times 10^{18} \mathrm{~cm}^{-3} \mathrm{Si}$ implant, the measured resistivity in the crater region is similar to the value measured in the surrounding AA inner ring area. It is known that in GaAs epitaxial films grown from a liquid-phase melt, the degree of amphoteric doping behavior of $\mathrm{Si}$ is a function of the $\mathrm{Si}$ doping concentration, with more amphoteric behavior occurring at high Si doping concentrations. ${ }^{11}$

The amphoteric behavior of $\mathrm{Si}$ in $\mathrm{InP}$ is minimal compared to in GaAs. ${ }^{2,12,13}$ In InP, as shown in Fig. 3(b), the measured resistivity in the crater is lower compared to that of the surrounding ring region. This probably is due to higher carrier mobility in the crater region compared to that in the $\mathrm{AA}$ inner ring region.

\section{X-ray diffraction results on Si-implanted GaAs and InP}

High resolution $\mathrm{x}$-ray diffraction measurements were made of $\mathrm{Si}^{+}$-implanted $\mathrm{GaAs}(001)$ and $\mathrm{InP}(001)$ using (004) reflection before and after laser shock. In the case of GaAs samples, one peak corresponding to pristine $\mathrm{GaAs}(004)$ at a $2 \theta$ angle of $66.048^{\circ}$ with a lattice parameter of $5.6533 \AA$ was observed before and after AA in the implanted sample (not shown). For the as-implanted GaAs, an additional satellite peak is observed at $65.75^{\circ}$ that corresponds to an expanded lattice parameter of $5.6807 \AA$. The implantation displaces Ga and As atoms from their equilibrium positions and the implant occupies interstitial positions in the GaAs, causing the lattice to expand and resulting in this low angle satellite peak. Upon laser pulse irradiation, this low angle satellite peak in the Si-implanted GaAs disappears and a new satellite peak appears at the high angle side (in the crater and near crater AA regions). The low angle and the high angle satellite peaks are distinct and are generated by different types of defects. When the x-ray scans were taken far from the crater region toward the center of the crater region the satellite peak shifted from the low angle side of the pristine GaAs peak to the high angle side. ${ }^{14}$ At the center of the crater region, the peak moved to $2 \theta=66.250^{\circ}$, corresponding to a lattice parameter of $5.6427 \AA$. The strain was maximal at the center of the crater region and showed a gradual reduction up to $4 \mathrm{~mm}$ from its center. Thus, the effective annealed region due to AA was limited to only a radius of $\sim 4 \mathrm{~mm}$, which roughly agrees with the electrical resistivity data presented in Fig. 


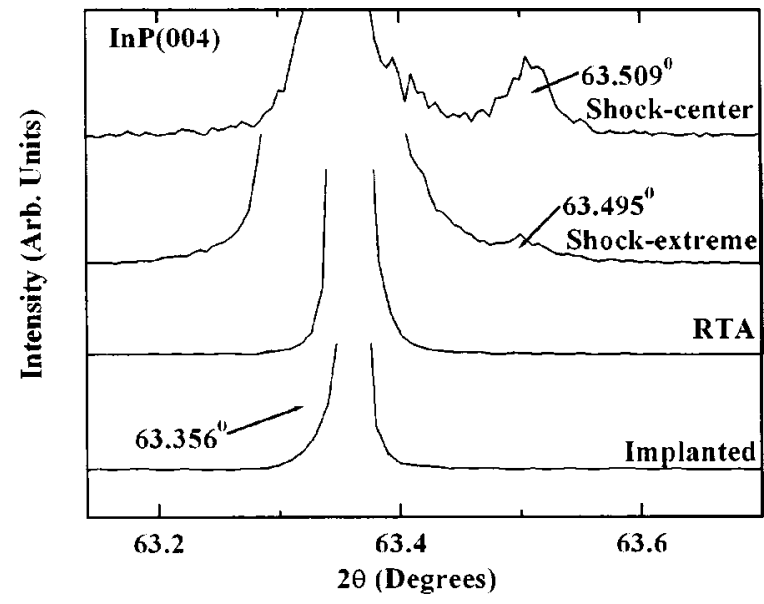

FIG. 4. High resolution $\mathrm{x}$-ray diffraction scans of pristine $\mathrm{InP}$ and Siimplanted InP before and after laser-pulse athermal annealing.

3(a). The presence of residual compressive strain was correlated with the reduction in resistivity for the same region. In the case of the RTA sample, the satellite peak was not observed, indicating that the damage induced by Si implantation was annealed out and that the Si goes substitutionally into the GaAs lattice.

In contrast to the GaAs sample, the as-implanted InP sample in Fig. 4 shows only one peak at $2 \theta=66.356^{\circ}$ corresponding to pristine $\mathrm{InP}(004)$. However, the AA sample shows an additional peak on the high angle side of the main peak. This additional peak was observed far from the crater region as well as in the crater region. The position of the peak changed from $2 \theta=63.495^{\circ}$ to $63.509^{\circ}$ because the scans were measured far from the crater region to the center of the crater region. In the case of the RTA sample of InP, only one peak at an angle of $2 \theta=63.356^{\circ}$ was observed which coincided with the position of a pristine InP sample.

\section{E. Discussion}

The laser intensity in the irradiated spot is sufficiently high $\left(>100 \mathrm{~J} / \mathrm{cm}^{2}\right)$ to produce hot plasma that streams away from the irradiated spot at high velocity, thereby launching multimode acoustical stress waves and shock waves into the wafer. ${ }^{3,8,15-17}$ The laser plasma is nonone dimensional in pulsed laser irradiations of long duration. ${ }^{15}$ For long pulsed laser irradiations, the plasma expansion becomes three dimensional at distances comparable to the laser spot diameter. The shock wave generated by the hot plasma is sustained until the pressure at the ablation front, which follows the shock, begins to drop rapidly at termination of the laser pulse. At this time rarefaction will propagate from the ablation front, eventually overtaking the shock wave and resulting in a decrease in shock pressure. ${ }^{15}$ In the present work the $\mathrm{x}$-ray topography study of the athermally annealed InP wafer has shown circular fringe-shaped regions with a decrease in intensity away from the laser spot. This provides evidence of the propagation of mechanical energy with a decrease in amplitude away from the laser spot. Lighter fringe regions were observed outside the effectively annealed regions of low electrical resistivity. This indicates that a certain amount of annealing that is sufficient to repair part of the lattice damage but not enough to activate the implanted dopant effectively has taken place at regions far from the laser spot center.

Commercial application of the laser-pulse athermal annealing technique demands the propagation of mechanical energy over the entire wafer by exposing the center of the wafer to a high-energy laser pulse. In this study, effective annealing is observed only in regions several $\mathrm{mm}$ from the laser spot. Optimization of this annealing technique to achieve effective annealing of implant damage and effective implant electrical activation at locations several centimeters away from the pulsed laser irradiation spot is being investigated.

\section{SUMMARY}

Athermal annealing of implanted GaAs and InP is possible by exposing the implanted face of the crystal to a highenergy laser pulse focused onto an $\sim 2 \mathrm{~mm}$ diam spot. The laser energy is absorbed by plasma above the sample surface. When the plasma gets very hot, it expands rapidly, generating backpressure which launches mechanical energy into the regions surrounding the laser exposed spot. This mechanical energy anneals implantation-induced lattice damage and drives the implant into the electrically active substitutional lattice sites at locations away from the irradiated spot, although this annealing effect is reduced at distances farther from the irradiated spot. For a laser pulse of $\sim 4 \mathrm{~J}$ energy and $\sim 35 \mathrm{~ns}$ duration, the annealed unexposed area is limited to an $\sim 8-12 \mathrm{~mm}$ outer diam ring-shaped zone surrounding the exposed $2 \mathrm{~mm}$ diam spot. No redistribution of the implant is observed at the implant tail in the athermally annealed region. Electrical resistivities measured on the athermal annealed region for $1 \times 10^{19} \mathrm{~cm}^{-3}$ Si-implanted GaAs are $\sim 1$ $\times 10^{-3} \Omega \mathrm{cm}$, which are similar to the values measured in the halogen-lamp rapid thermally annealed material. For the annealing conditions used in this study, although substitutional lattice recovery took place, a satellite peak in the x-ray diffraction measurements is observed in the athermally annealed high-dose Si-implanted material compared to in the pristine material, indicating the presence of some residual implant damage. Optimization of the laser power, spot size and pulse duration may cause more effective annealing at distances much farther from the irradiated spot than what was observed in this study.

\section{ACKNOWLEDGMENTS}

Work at George Mason University was supported by the National Science Foundation under Grant No. ECS-0079363. The work of two of the authors (R. F. and J. G.) was supported by a grant from the Office of Naval Research. The authors thank Peter Chi of the National Institute for Standards and Technology for help with the SIMS measurements.

\footnotetext{
${ }^{1}$ J. P. de Souza and D. K. Sadana, in Handbook on Semiconductors, edited by S. Mahajan (Elsevier, Amsterdam, 1994), Vol. 3, Chap. 27.

${ }^{2}$ M. V. Rao, IEEE Trans. Electron Devices 40, 1053 (1993).

${ }^{3}$ J. Grun et al., Phys. Rev. Lett. 78, 1584 (1997).

${ }^{4}$ D. W. Donnelly, B. C. Covington, J. Grun, C. A. Hoffman, J. R. Meyer, C.
} 
K. Manka, O. Glembocki, S. B. Qadri, and E. F. Skelton, Appl. Phys. Lett. 71, 680 (1997).

${ }^{5}$ J. Grun, R. P. Fischer, M. Peckerar, C. L. Felix, B. Covington, W. J. DeSisto, D. W. Donnelly, A. Ting, and C. K. Manka, Appl. Phys. Lett. 77, 1997 (2000).

${ }^{6}$ D. W. Donnelly, B. Covington, J. Grun, R. P. Fischer, M. Peckerar, and C. L. Felix, Appl. Phys. Lett. 78, 2000 (2001).

${ }^{7}$ L. D. Landau and E. M. Lifshitz, Fluid Mechanics, 2nd ed. (Butterworth Heinemann London, 1995), p. 192.

${ }^{8} \mathrm{~J}$. Grun et al., Proceedings of the Electrochemical Society (The Electrochemical Society, Pennington, NJ, 2000), Vol. 2000-9, p. 107.

${ }^{9}$ K. S. Jones, E. L. Allen, H. G. Robinson, D. A. Stevenson, M. D. Deal, and J. D. Plummer, J. Appl. Phys. 70, 6790 (1991).
${ }^{10}$ J. P. de Souza and D. K. Sadana, IEEE Trans. Electron Devices 39, 166 (1992).

${ }^{11}$ I. Teramoto, J. Phys. Chem. Solids 33, 2089 (1972).

${ }^{12}$ S. J. Pearton and U. K. Chakrabarti, in Indium Phosphide and Related Materials: Processing, Technology, and Devices, edited by A. Katz (Artech, Norwood, MA, 1992), Chap. 7.

${ }^{13}$ G. G. Baumann, K. W. Benz, and M. H. Pilkuhn, J. Electrochem. Soc. 123, 1232 (1976)

${ }^{14}$ S. B. Qadri et al. (unpublished).

${ }^{15}$ R. J. Trainor and Y. T. Lee, Phys. Fluids 25, 1898 (1982).

${ }^{16}$ B. Meyer and G. Thiell, Phys. Fluids 27, 302 (1984).

${ }^{17}$ J. Grun, R. Stellingwerf, and B. H. Ripin, Phys. Fluids 29, 3390 (1986). 